\title{
Efecto de la correspondencia entre descripciones de contingencias y contingencias sobre la conducta de elección bajo paradigma de autocontrol
}

\author{
Yamile Andrea Gómez Delgado*, Diana Marcela Muñoz Rosero, Edwin Gerardo Luna Tascón y Juan Carlos Benavides Martínez \\ Grupo de Investigación Psicología y Salud \\ Universidad de Nariño, Colombia
}

Recibido, agosto 3/2016

Concepto de evaluación, febrero 3/2017

Aceptado, mayo 16/2017
Referencia: Gómez-Delgado, Y.A., Muñoz Rosero, D.M., Luna Tascón, E.G. \& Benavides Martínez, J.C.(2017). Efecto de la correspondencia entre descripciones de contingencias y contingencias, sobre la conducta de elección bajo paradigma de autocontrol. Acta colombiana de Psicología, 20(2), 227-239. doi: http://www.dx.doi.org/10.14718/ ACP.2017.20.2.11

Resumen

\begin{abstract}
En este trabajo se analiza el efecto de la historia de correspondencia entre las descripciones de contingencias y las contingencias sobre la conducta de elección bajo paradigma de autocontrol en 85 participantes de entre 10 y 11 años de edad, por medio de dos experimentos intrasujeto, uno realizado en contexto de laboratorio y otro en una situación natural simulada. Los resultados indican que es posible afectar la conducta de elección entre un reforzador inmediato — de menor magnitud — y otro demorado - de mayor proporción - en favor de la segunda alternativa a partir de una historia experimental de correspondencia entre las descripciones de contingencias y las contingencias. En ambos experimentos, la mayoría de participantes eligió el reforzador demorado después de experimentar los ensayos de correspondencia entre descripciones y contingencias, y el reforzador inmediato después de enfrentar los ensayos de correspondencia ausente pese a que las situaciones de entrenamiento fueron formal y funcionalmente diferentes en relación con la tarea establecida para evaluar la conducta de elección. Los resultados se analizan a la luz de la Teoría de los Marcos Relacionales, específicamente en relación con la alteración de las funciones del lenguaje.

Palabras clave: Autocontrol, conducta de elección, lenguaje, claves contextuales, descripciones de contingencias.
\end{abstract}

\section{The effect of correspondence between descriptions of contingencies and contingencies on the conduct of election under the self-control paradigm}

\begin{abstract}
In this paper, the effect of correspondence history between contingency and contingency descriptions on the behavior of choice under the self-control paradigm is analyzed in 85 participants between the ages of 10 and 11, through two intrasubject experiments, one carried out in a laboratory context and another in a simulated natural situation. The results indicate that it is possible to affect the behavior of choice between an immediate reinforcer of lesser magnitude and a delayed one of greater magnitude in favor of the second alternative, from an experimental history of correspondence between descriptions of contingencies and contingencies. In both experiments, most of participants chose the delayed reinforcer after undergoing correspondence tests between descriptions and contingencies, and the immediate reinforcer after facing the correspondence absent trials, even though the training situations were formally and functionally different in relation with the task established to evaluate the conduct of choice. The results are analyzed in light of the "Relational Frame Theory", specifically, in relation with the alteration of the functions of the language.

Key words: Self-control, choice behavior, language, contextual keys, contingency descriptions.
\end{abstract}

\footnotetext{
* Universidad de Nariño, Departamento de Psicología, Calle 18 Carrera 50-02, Ciudad Universitaria Torobajo, (2)7315444, andreagomezdelgado21@gmail.com
} 


\title{
Efeito da correspondência entre descriçóes de contingências e contingências sobre o comportamento de escolha sob o paradigma de autocontrole
}

\author{
Resumo
}

\begin{abstract}
Neste trabalho, analisa-se o efeito da história de correspondência entre as descrições de contingências e contingências sobre o comportamento de escolha sob o paradigma de autocontrole com 85 participantes entre 10 e 11 anos de idade, por meio de duas experiências intrassujeito, uma realizada em contexto de laboratório, e outra, numa situação natural simulada. Os resultados indicam que é possível afetar o comportamento de escolha entre um reforçador imediato — de menor magnitude- e outro demorado — de maior proporção - em favor da segunda alternativa a partir de uma história experimental de correspondência entre as descrições de contingências e contingências. Em ambas as experiências, a maioria de participantes escolheu o reforçador demorado depois de experimentar os ensaios de correspondência entre descrições e contingências, e o reforçador imediato depois de enfrentar os ensaios de correspondência ausente, embora as situações de treinamento tenham sido formal e funcionalmente diferentes com relação à tarefa estabelecida para avaliar o comportamento de escolha. Os resultados foram analisados à luz da Teoria das Molduras Relacionais, especificamente quanto à alteração das funções da linguagem.

Palavras-chave: Autocontrole, chaves contextuais, comportamento de escolha, descrições de contingências, linguagem.
\end{abstract}

\section{INTRODUCCIÓN}

La tendencia a comportarse en función de consecuencias demoradas - lo que comúnmente recibe el nombre de autocontrol - es aprendida y consiste específicamente en la elección de la alternativa más óptima a largo plazo, en términos de calidad y magnitud del reforzamiento, o de evitación de la estimulación aversiva (American Psychological Association, 2012; Martin \& Pearl, 2006; Montgomery, 2008; Skinner, 1974, citado por Gómez \& Luciano, 1991; Theler \& Shefrin, 1981).

En un informe publicado por la American Psychology Association (2012) se menciona que el autocontrol se relaciona con estilos de vida saludables, tales como practicar una rutina de ejercicio regular, llevar una dieta sana, evidenciar alto desempeño académico, bajos índices de abuso de sustancias y tener mayor seguridad financiera, entre otros. Asimismo, se hace alusión a una variedad de problemáticas relevantes socialmente que se asocian a la tendencia contraria: comportarse en función de consecuencias inmediatas aun cuando ello implique experimentar situaciones desagradables a futuro, como la obesidad (American Psychology Association, 2012; Organización Mundial de la Salud, 2012), el consumo de cigarrillo (Organización Mundial de la Salud \& Ministerio de Sanidad y Consumo, 2006), el consumo de drogas ilícitas (Naciones Unidas, 2012; Tibbetts \& Whittimore, 2002 citados por Cáceres, Salazar, Varela $\&$ Tovar, 2006), el uso excesivo de internet — relacionado con la disminución del tiempo destinado a la realización de actividades académicas y laborales- (García, 2008) y la contaminación ambiental (Corral, 2006; Organización Mundial de la Salud, 2010).

La relevancia de esta variable ha llevado a diferentes autores a plantear estrategias que favorecen el desarrollo de comportamientos autocontrolados. Inicialmente, Skinner (1974, citado por Montgomery, 2008) hizo referencia a los siguientes procedimientos: 1) restricción y ayuda física; 2) manipulación de estados de privación y saciedad; 3) manipulación de estados emocionales; 4) uso de estímulos aversivos; 5) uso de fármacos o estimulantes; 6) autorreforzamiento o autoextinción; 7) autocastigo de respuestas indeseables; y 8) respuestas incompatibles.

Posteriormente, a partir de diferentes investigaciones se ha llegado a diferenciar tres procedimientos útiles para incrementar la probabilidad de elegir la opción demorada en una situación de elección:

- El primero se denomina maximización de la respuesta por manipulación del intervalo de demora, el cual tiene que ver con la manipulación de la cantidad, la cualidad o la demora de reforzamiento (Dixon \& Cummings, 2001; Gómez \& Luciano, 1991; 2000). Con la aplicación de este procedimiento se ha encontrado que entre más cerca del final del intervalo de espera se presente la oportunidad de elegir entre un estímulo inmediato de menor magnitud, versus el establecido de mayor magnitud para el final del intervalo, mayor es la probabilidad de que se elija esperar (González, Ávila, Juárez \& Miranda, 2011). De igual forma, se ha observado que es posible incrementar el autocontrol mediante la repetición de ensayos de elección, incrementando de 
manera progresiva la demora para obtener el refuerzo de mayor magnitud (Dixon \& Cummings, 2001).

- El segundo es el uso de distractores o conductas alternativas, el cual propone que los distractores de los reforzadores inmediatos o la atención hacia otros estímulos facilitan la espera (Dixon, Rehfeldt \& Randich, 2003; Gómez \& Luciano, 1991). Es decir, la probabilidad de elegir la opción del reforzador demorado se incrementa cuando existe la posibilidad de realizar actividades alternas simultáneas al tiempo de espera (Dixon \& Cummings, 2001; Grosch \& Neuringer, 1981).

- Y el tercero tiene que ver con la formulación de autoinstrucciones, el autorreforzamiento, o su aplicación conjunta (O'Leary \& Duvey, 1979 citados en Gómez \& Luciano, 1991). Dentro de esta última categoría se encuentran los programas de correspondencia decirhacer, en los cuales el participante es entrenado para mostrar coherencia entre la descripción de lo que hará y lo que realmente hace luego de decirlo; en estos procedimientos no se requiere de ayudas como modelos o instrucciones de espera (Gómez \& Luciano, 2000).

Con relación a estos procedimientos, Gómez y Luciano (1991) observaron que con el aumento gradual de la demora, si bien se incrementa la elección del reforzador demorado de mayor magnitud en la situación entrenada, no se logra la transferencia a comportamientos no entrenados. A diferencia de esto, el procedimiento decir-hacer, además de generar un repertorio de espera, produce un repertorio para facilitarla (equivalente a los distractores). De igual forma, observaron que puede darse una generalización funcional del procedimiento decir-hacer a través de la similitud de instrucciones o de características contextuales -0 específicas - de una tarea a otra.

Este último grupo de procedimientos para generar comportamiento autocontrolado pone de manifiesto que el lenguaje constituye una variable relevante para el estudio del autocontrol en humanos (Benabou \& Tirole, 2004; Gómez, López \& Mesa, 2007; Gómez \& Luciano, 1991; 2000; Guevremont, Osnes \& Stokes, 1986; Luciano, 1992; Montgomery, 2008; Wilson \& Luciano, 2007).

El interés por el estudio del lenguaje desde la perspectiva conductual se remonta al primer abordaje sistemático del mismo —el realizado por Skinner (1981) —, donde además de caracterizarlo como un comportamiento cualquiera que se aprende a través de reforzamiento, se planteó el concepto de "discriminación condicional" como aspecto de importancia central para su comprensión. Este fenómeno hace referencia a una situación en la que la respuesta de un individuo ante un estímulo depende de otro $u$ otros estímulos que alteran las funciones del primero (Alós, Guerrero, Falla \& Amo, 2013; Hernández \& Sandoval, 2003). Los estudios de discriminación condicional, generaron un contexto pertinente para el descubrimiento y conceptualización de un procedimiento para el análisis experimental de la formación de categorías: la equivalencia de estímulos (Sidman, 1971; Zentall, Galizio \& Critchfield, 2002).

La emergencia de clases de estímulos equivalentes es un proceso conductual básico que ha permitido la explicación de respuestas relacionales que surgen sin entrenamiento directo, es decir, a partir de su vinculación - mutua o combinatoria - con otros estímulos (Hernández \& Sandoval, 2003). En los años 90 se propuso una aproximación analíticocomportamental al lenguaje y a la cognición denominada Teoría de los Marcos Relacionales (TMR), la cual aborda el surgimiento de relaciones entre estímulos no entrenadas directamente y respuestas relacionales arbitrariamente aplicables, a través de una terminología más genérica que se adapta no solo a las relaciones de equivalencia, sino también a las de no equivalencia, como la oposición, la distinción, la comparación, la temporalidad o la causalidad, entre otras (Barnes-Holmes, Rodríguez \& Whelan, 2005; Hayes, Barnes-Holmes \& Roche, 2001).

Desde la perspectiva de la TMR, el lenguaje es una respuesta operante que consiste en aprender a relacionar eventos con criterios arbitrariamente establecidos - no con base en sus propiedades formales-, a través de diversos ejemplos y situaciones que llevan a abstraer la clave contextual que los relaciona para aplicarla a eventos diferentes y novedosos (Valdivia \& Luciano, 2006). Así, la respuesta de un participante ante estímulos nunca antes vistos se presenta en función de claves contextuales que delimitan marcos de relación y que pueden ser de equivalencia (es igual a), de diferencia (es diferente de), de comparación (más que o menos que), de temporalidad (antes de o después de) o de causalidad (si, entonces), entre otros, los cuales definen la relación entre eventos (Hayes et al., 2001; Luciano, Gómez, Hernández \& Cabello, 2001).

Desde esta perspectiva, la descripción de una contingencia afecta a los eventos a los que se refiere debido la posibilidad de que se transfieran o transformen las funciones entre los estímulos - palabras y eventos referidos - que hacen parte de un marco relacional, en función de los marcos relacionales a los que pertenecen (Luciano et al., 2001). En relación con el comportamiento autocontrolado, la adquisición de un repertorio verbal consolidado le permite a los seres humanos, entre otras cosas, contactar funcionalmente con las propiedades apetitivas de la elección que lleva al reforzador demorado —o las aversivas vinculadas - con la elección del reforzador inmediato, aun cuando no estén presentes formalmente en la situación con la que se interactúa.

De acuerdo con Wilson y Luciano (2007), el control verbal sobre una acción en un contexto particular sería un 
caso de relación arbitraria entre comportamiento verbal y no verbal. Según las autoras, este tipo de descripciones llegan a tener un control discriminativo cuando en la historia del individuo se han generado interacciones numerosas y variadas para formar marcos relacionales pertinentes. De este modo, las interacciones que un individuo tiene - desde su infancia - con otros hablantes son determinantes para que se genere el repertorio de regulación verbal.

En el caso de la conducta de elección bajo paradigma de autocontrol, el comportamiento verbal permite contactar con consecuencias que solo estarán disponibles a largo plazo - o que son poco probables - mediante la vinculación verbal de la situación actual con eventos que no están presentes en la misma dimensión espacio-temporal, además de facilitar el aprendizaje de comportamientos sin que sea necesario exponerse a consecuencias aversivas (Törneke, Luciano \& Valdivia 2008).

Evidencia de este fenómeno se muestra en el estudio de Binder, Dixon y Ghezzi (2000) realizado con niños con edades comprendidas entre los 3 y 5 años que habían sido diagnosticados con trastorno de atención e hiperactividad; específicamente, los resultados mostraron que cuando a los participantes se les propuso en un primer momento la opción entre un reforzador inmediato de menor magnitud y un reforzador demorado pero de mayor proporción, todos eligieron el reforzador más pequeño, sin embargo, cuando se introdujeron ligeros retrasos para obtener un reforzador más grande junto con la verbalización de la autorregla "Si espero más, tendré el más grande", todos los participantes demostraron autocontrol. En esta misma línea, Ruizy Gómez (2016), en un estudio realizado con personas con edades entre los 12 y 15 años de edad, observaron que a medida que estos aprenden a responder a diferentes verbalizaciones, se incrementa la frecuencia de comportamientos en función de consecuencias demoradas, los cuales resultan indispensables en situaciones en las que deben enfocarse en tareas monótonas que no representan una recompensa inmediata, pero que serán útiles a futuro.

Con base en lo expuesto, el presente trabajo se realizó bajo la hipótesis de que la correspondencia - o ausencia de esta - entre descripciones de contingencias y las contingencias mediadas por otros individuos durante la infancia afectan el ajuste ante descripciones de contingencias que implican comportarse en función de consecuencias demoradas de mayor magnitud, es decir, sobre la conducta autocontrolada. Se entiende por correspondencia entre descripciones de contingencias y contingencias cuando la verbalización de los elementos de la contingencia a experimentar coincide o se relaciona directamente con lo que la persona efectivamente experimenta; mientras que por correspondencia ausente se entiende la inconsistencia entre la verbalización de la contingencia y la experiencia de la misma.

\section{MÉTODO}

Se realizaron dos experimentos para analizar el efecto diferencial de una historia de correspondencia o ausencia de correspondencia entre descripciones de contingencias y contingencias sobre la elección de reforzadores inmediatos o postergados. El primero fue realizado en una situación de laboratorio y el segundo en una situación natural simulada.

\section{EXPERIMENTO I}

\section{Participantes}

En el primer experimento participaron 12 estudiantes de grado quinto de una institución educativa estatal, elegidos de manera aleatoria, con edades comprendidas entre los 10 y 11 años. La elección de los participantes se hizo teniendo en cuenta el reporte brindado por el acudiente y el docente orientador de la institución, el cual permitió descartar variables psicológicas o médicas que pudieran afectar el desempeño del participante durante la investigación. Es importante señalar que todos ellos carecían de experiencia en la tarea experimental.

\section{Aparatos y situación experimental}

Las sesiones experimentales se llevaron a cabo en un aula del establecimiento educativo equipada con un computador de mesa Hewlett Packard Intel Core I5, una cámara de video, un dispensador de reforzadores (monedas de 50 COP, lo que equivale a .01730 USD), una silla situada frente al monitor para el participante, y una silla para el experimentador ubicada a una distancia de aproximadamente dos metros a la derecha del niño.

\section{Diseño}

En este estudio se empleó un diseño intrasujeto, y los participantes fueron asignados a dos condiciones experimentales de manera aleatoria, tal como se observa en la Tabla 1.

Después de cada bloque de ensayos se midió la variable autocontrol por medio de una tarea de elección. Cabe mencionar que el diseño utilizado no comprende línea de base, ya que realizar una medida previa de la conducta de elección podría afectar las subsecuentes evaluaciones, dados los efectos del aprendizaje (Kerlinger \& Lee, 2002). 
Tabla 1

Condiciones experimentales

\begin{tabular}{ccc}
\hline $\begin{array}{c}\text { Condiciones } \\
\text { experimentales }\end{array}$ & Primer bloque de ensayos & Segundo bloque de ensayos \\
\hline 1 (seis participantes). & $\begin{array}{c}\text { Correspondencia ausente entre descripciones de } \\
\text { contingencias y contingencias. }\end{array}$ & $\begin{array}{c}\text { Correspondencia entre descripciones de contin- } \\
\text { gencias y contingencias. }\end{array}$ \\
2 (seis participantes). & Correspondencia entre descripciones de contin- \\
& gencias y contingencias. & $\begin{array}{c}\text { Correspondencia ausente entre descripciones de } \\
\text { contingencias y contingencias. }\end{array}$ \\
\hline
\end{tabular}

\section{Procedimiento}

Cada participante ingresaba al aula de manera individual y tomaba asiento frente al monitor. De esta manera, el investigador procedía a dar la instrucción general de la actividad:

A continuación vas a realizar un juego. En la pantalla del computador aparecerán las instrucciones, yo voy a estar aquí por si tienes alguna duda. Debes tener en cuenta que yo no te voy a dar una calificación por lo que vas a hacer ahora, estamos en confianza, si tienes alguna duda, puedes preguntarme, ¿está bien? Ahora puedes comenzar a leer.

El investigador permanecía dentro del aula con el fin verificar que el participante leyera la instrucción completa, aclarar posibles dudas y contabilizar el tiempo de ejecución en la tarea planteada para evaluar el autocontrol.

Todos los participantes fueron expuestos a las siguientes fases: a) ensayos de correspondencia, b) tarea de elección, c) ensayos de correspondencia ausente, y d) tarea de elección. La diferencia entre las dos condiciones experimentales fue el orden en que se presentaron los bloques de ensayos, tal como se muestra en la sección de diseño. Para las dos condiciones experimentales se utilizó un procedimiento de discriminación condicional conformado por 14 ensayos de correspondencia entre descripciones y contingencias, y 14 ensayos de correspondencia ausente, todos presentados con el programa Microsoft Office Power Point 2007.

Cada ensayo consistió en la presentación de una descripción de contingencias ubicada en la parte superior de la pantalla y diferentes estímulos acordes con la descripción: números, figuras geométricas, animales y objetos extraídos de la Escala de Inteligencia Revisada para el Nivel Escolar del WISC-R de 1981; esto para garantizar el conocimiento de dichos estímulos por parte de los participantes.

En el bloque de correspondencia, la descripción de la contingencia correspondía con la contingencia experimentada en todos los ensayos. De esta manera, cada vez que el participante elegía la opción correcta, en la pantalla aparecía un mensaje que le indicaba su acierto; y al accionar el dispensador, salía una moneda de 50 pesos (COP). Por el contrario, cuando elegía la opción incorrecta, aparecía una pantalla en blanco, y transcurridos cinco segundos, se presentaba automáticamente el mismo ensayo.

En el bloque de correspondencia ausente, la descripción no correspondía con la contingencia en ninguno de los ensayos; es decir, aunque la descripción le informaba al participante que recibiría una moneda de 50 pesos (COP) por una ejecución, al accionar el dispensador no se suministraba el reforzador.

La instrucción inicial para cada bloque de ensayos se presentó por medio de tres diapositivas para mayor comprensión del participante. Cada una de estas incluía un ejemplo gráfico de los estímulos, la ejecución requerida y las consecuencias:

Pon mucha atención: en la pantalla aparecerán unas figuras. Tú debes hacer clic en la figura que te indique la instrucción [Diapositiva 1]. Si lo haces de manera correcta, aparecerá una imagen de una moneda de 50 pesos como la que aparece abajo de la pantalla [Diapositiva 2]. Cuando salga la imagen de la moneda, ve al dispensador y coge únicamente la ficha que el computador te dice y el dispensador te dará una moneda de 50 pesos [Diapositiva 3].

Cuando el participante terminaba de leer la instrucción, se presentaba una diapositiva con un ejemplo y el investigador indicaba la ubicación del dispensador y su modo de uso. Para garantizar que el niño comprendiera la instrucción, aparecía una diapositiva en la que se planteaban dos opciones: leer nuevamente la instrucción o dar inicio al juego. Al dar clic en inicio, se presentaba el bloque de ensayos de correspondencia o ausencia de correspondencia dependiendo de la condición experimental. La diferencia entre las dos condiciones era la pertinencia de la descripción de la contingencia (es decir, si describe lo que sucedía realmente o no), ya que en la condición de correspondencia el participante obtenía la moneda de 50 pesos (COP) al accionar el dispensador, mientras que en la condición de correspondencia ausente esto no se cumplía. 
Al completar los 14 ensayos de cada bloque aparecía una diapositiva que planteaba una situación de elección para evaluar la variable autocontrol. La tarea del participante en esta fase era dar clic sobre la opción que prefiriera: finalizar la actividad, evitando el esfuerzo requerido para realizar una tarea monótona y larga (reforzador inmediato de baja magnitud); o transcribir 500 letras que aparecían en la pantalla para obtener 600 pesos (.2053 USD), lo que se constituyó como el reforzador demorado. Las opciones se presentaron como se muestra a continuación:

(a) "quiero terminar la actividad en este momento", o (b) "Quiero copiar las 500 letras que están en la parte de debajo de pantalla en la hoja que está sobre la mesa para ganar 600 pesos". Si eliges esta opción, al terminar de copiar todas las letras coge la ficha número $15 \mathrm{y}$ el dispensador te dará tus 600 pesos.

Cuando el participante elegía la primera opción, aparecía una diapositiva que indicaba que la actividad había finalizado y salía del aula; pero cuando elegía la segunda opción, una diapositiva repetía la instrucción sobre la transcripción de las letras.

En el momento en que el participante comenzaba a transcribir las letras en la hoja dispuesta sobre la mesa, se activaba un cronómetro para contabilizar el tiempo de ejecución. Cada cuatro minutos, el experimentador se encargaba de preguntar al participante si deseaba continuar o suspender la tarea, recordando que podía tomar la opción que prefiriera. Esto se hizo con el objetivo de recrear una situación similar a lo que sucede en la vida cotidiana, cuando la persona puede optar por el reforzador inmediato en cualquier momento (dependiendo de la disponibilidad). La instrucción elaborada para instigar al participante fue: "¿Deseas continuar copiando las letras o prefieres dejar de hacerlo? Puedes elegir la opción que prefieras".

El experimentador acompañaba al participante hasta que terminaba la actividad, y, posteriormente, el participante se dirigía a una sala contigua donde permanecía con otro experimentador durante 10 minutos. Transcurridos los 10 minutos de descanso, el participante regresaba al aula y el procedimiento se repetía con el segundo bloque de ensayos.

Concluido el estudio, el investigador le explicó a cada participante la finalidad del experimento y se instruyó frente a la importancia de seguir las instrucciones que provengan de fuentes confiables como sus padres y docentes.

\section{RESULTADOS}

Los resultados del experimento I indican que la elección de los participantes entre un reforzador demorado de mayor magnitud y un reforzador inmediato de menor magnitud se ve afectada después de experimentar correspondencia o ausencia de correspondencia entre las descripciones de contingencias proporcionadas y las contingencias que estas describen.

Entre los seis participantes que experimentaron inicialmente los bloques de correspondencia ausente y después los bloques de correspondencia, tres eligieron el reforzador inmediato de menor magnitud en la primera situación (P2, P3 y P6) y tres optaron por transcribir las 500 letras para obtener el reforzador demorado de mayor magnitud (P1, P4 y P5). Sin embargo, dos de los participantes que eligieron esta última opción (P1 y P4) abandonaron la actividad a los cuatro minutos correspondientes a la presentación del primer instigador, y solo uno de los participantes finalizó la transcripción de las letras (P5) después de 28 minutos.

Al pasar a los bloques de correspondencia entre descripciones de contingencias y contingencias, uno de los participantes eligió el reforzador inmediato de menor magnitud (P2) y cinco eligieron el reforzador demorado de mayor proporción (P1, P3, P4, P5 y P6). De estos últimos, tres (P4, P5, y P6) culminaron la tarea en un promedio de 30.4 minutos, a pesar de la presentación de los instigadores; uno escribió más de la mitad del contenido, abandonando la tarea a los 16 minutos de iniciada la ejecución (P1); y otro abandonó la actividad transcurridos 4 minutos (P3). En la Figura 1 se muestra la cantidad de letras transcritas por los participantes en cada bloque.

Por otra parte, los seis participantes que experimentaron primero los bloques de correspondencia y posteriormente los bloques de correspondencia ausente eligieron el reforzador demorado en el primer bloque; cinco de ellos culminaron la transcripción de letras en aproximadamente 30 minutos (P7, P9, P10, P11 y P12) y uno realizó más de la mitad de la transcripción (P8), abandonando la tarea a los 15 minutos del inicio de la ejecución (antes de la presentación del cuarto instigador).

Posteriormente, en los bloques de correspondencia ausente, cuatro participantes eligieron el reforzador inmediato (P8, P9, P11 y P12) y dos optaron por el reforzador demorado de mayor magnitud (P7 y P10); uno de ellos logró culminar la tarea transcurridos 24 minutos (P10) y el otro desistió a los 4 minutos después de la presentación del primer instigador. En la figura 2 se muestra la cantidad de letras transcritas por los participantes en cada bloque de esta condición experimental.

En síntesis, es posible observar que cuando los participantes experimentaron ensayos de correspondencia entre descripciones de contingencias y contingencias de manera previa a la situación de elección bajo paradigma de autocontrol, presentaron mayor tendencia a elegir el reforzador demorado de mayor magnitud, es decir, a mostrar comportamiento 


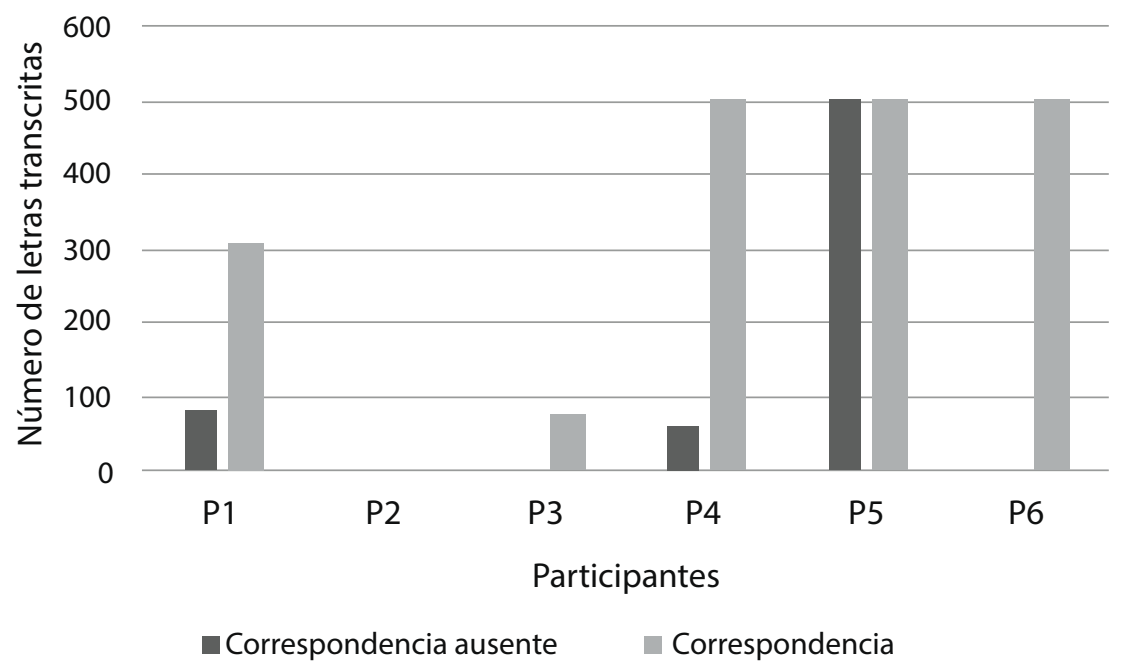

Figura 1. Conducta de elección en la condición experimental de correspondencia ausente y correspondencia.

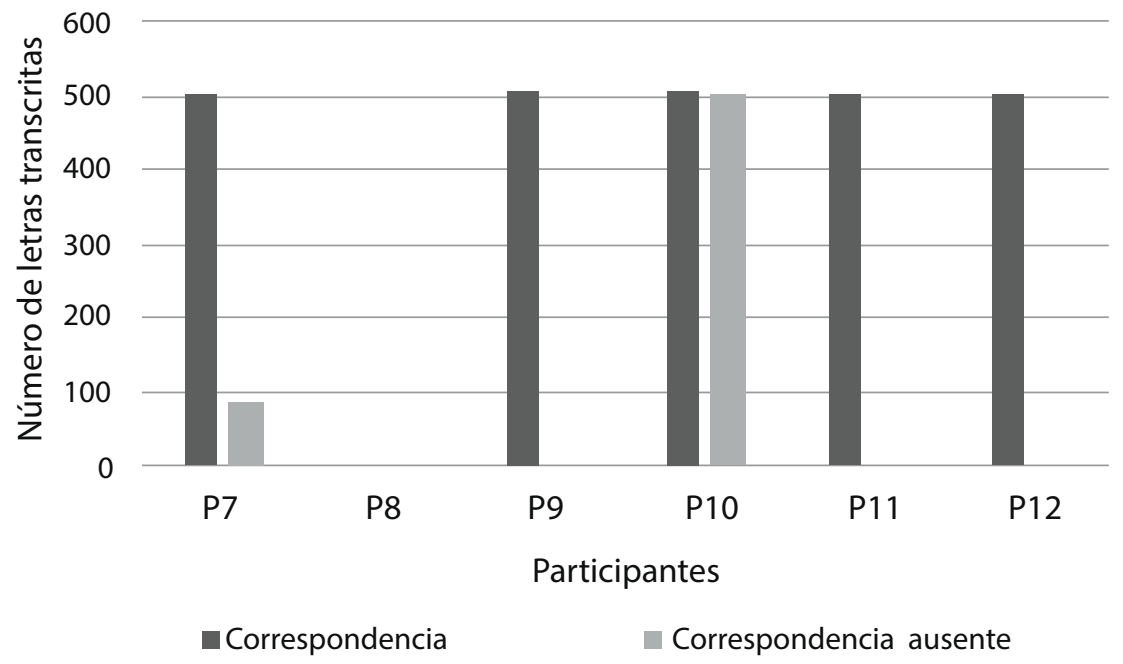

Figura 2. Conducta de elección en la condición experimental de correspondencia y correspondencia ausente.

autocontrolado. En contraste, al experimentar los ensayos de correspondencia ausente, los participantes evidenciaron mayor tendencia a elegir el reforzador inmediato de menor magnitud. Este efecto se observó en la medición de las dos condiciones experimentales.

\section{EXPERIMENTO II}

\section{Participantes}

En el segundo experimento participaron 63 estudiantes de quinto grado con edades comprendidas entre 10 y 11 años y pertenecientes a una institución educativa estatal.
Los participantes fueron elegidos teniendo en cuenta el reporte brindado por el acudiente y el docente orientador de la institución; el cual permitió descartar variables psicológicas o médicas que pudieran afectar el desempeño del participante durante la investigación. Adicionalmente, todos los participantes carecían de experiencia en la tarea experimental y hacían parte de dos cursos elegidos aleatoriamente entre 13 cursos de quinto de primaria de la institución educativa.

\section{Aparatos y situación experimental}

El experimento se realizó en dos sesiones de 45 minutos cada una, las cuales se llevaron a cabo en un aula de la institución educativa equipada con una mesa, sillas y una 
cámara de video. Durante la aplicación del experimento no estuvieron presentes otras personas además de los participantes y el investigador.

\section{Diseño}

En este experimento se empleó un diseño intrasujeto aplicado en una situación natural simulada. En la Tabla 2 se presentan las condiciones experimentales.

De manera posterior a cada bloque de ensayos se realizó la evaluación de la variable autocontrol por medio de una tarea de elección.

\section{Procedimiento}

Una semana antes del desarrollo del experimento, el equipo de investigación solicitó la autorización para realizar el estudio a la institución y a los encargados legales de los participantes. Junto con el consentimiento informado, los acudientes diligenciaron una lista de chequeo que permitió obtener información acerca de aspectos psicológicos o médicos que pudieran impedir la participación del niño. Adicionalmente, se solicitó a los padres de familia que se abstuvieran de dar recomendaciones a sus hijos acerca de cómo comportarse en el experimento.

Se desarrollaron dos situaciones naturales simuladas como actividades dentro del horario escolar de los participantes en las dos primeras horas de clase (entre 7:00 a. m. y 9:00 a. m.). Las tareas fueron presentadas por una fuente humana (experimentador); cada una era resuelta por uno de los participantes; y las consecuencias de esta eran experimentadas por todos los niños. Las actividades fueron diseñadas de tal forma que realizarlas no implicara un trabajo arduo para el participante, y garantizando una respuesta acertada en todos los casos. Se estructuraron en total cinco tareas que se mantuvieron para las dos condiciones experimentales. Y se establecieron reforzadores como dulces y tiempo de descanso.

Inicialmente, se administró uno de los dos bloques de ensayos (es decir, correspondencia o ausencia de correspondencia entre descripciones de contingencias y contingencias) y después de medir el efecto en la conducta de elección bajo paradigma de autocontrol, se aplicó el bloque restante. De los 73 participantes, 37 experimentaron los ensayos en el orden correspondencia-ausencia de correspondencia, y 36 en el orden ausencia de correspondencia-correspondencia.

En los bloques de correspondencia entre descripciones y contingencias, los participantes se enfrentaron a cinco tareas presentadas por un experimentador (fuente). En cada tarea, la fuente suministró una descripción de contingencia que mencionaba una situación estímulo, una respuesta y unas consecuencias. Dicha descripción era seguida por uno de los participantes, y las consecuencias (reforzantes) por realizar correctamente la conducta las experimentaba todo el grupo.

Por ejemplo, para la tarea 1 el experimentador elegía al azar a un participante del grupo, lo llamaba a pasar adelante y daba la siguiente descripción: "Mira este dado, hace un momento lo lancé y saqué dos, si tú lo lanzas y sacas más de dos, les daré a todos un chocolate".

Cuando el participante lograba realizar la actividad como se planteaba, el experimentador proporcionaba las consecuencias descritas a todo el grupo. Si el participante fallaba, se le daba otra oportunidad, hasta que lograra cumplir el criterio de la tarea. Las cuatro tareas siguientes se realizaban de manera similar dentro de los 45 minutos estipulados para la sesión.

En los bloques de correspondencia ausente se conservó la fuente y las tareas de la primera sesión, sin embargo, aun cuando el participante cumplía con el criterio de acierto mencionado en la descripción de la contingencia, no se administraba la consecuencia descrita. Por ejemplo, para la tarea 2, el experimentador elegía al azar a un participante del grupo, lo llamaba a pasar adelante y mencionaba la siguiente descripción de contingencia: "Mira estas tarjetas, del otro lado son de diferentes colores, lo que tú debes hacer es señalar una de ellas, si esa tarjeta por el reverso es de color amarillo, los dejo salir a jugar a todos durante 5 minutos".

Cuando el participante lograba realizar la actividad como se planteaba, el experimentador no proporcionaba las consecuencias descritas, debido a que se encontraban en el bloque de correspondencia ausente, diciendo lo siguiente: "Bien sacaste la tarjeta amarilla, pero no los voy a dejar salir a jugar". Si el participante fallaba, se le daba otra oportunidad hasta que lograba cumplir el criterio de la

Tabla 2

Condiciones experimentales

\begin{tabular}{ccc}
\hline Condiciones experimentales & Primer bloque de ensayos & Segundo bloque de ensayos \\
\hline 1 (37 participantes). & $\begin{array}{c}\text { Correspondencia entre descripciones de con- } \\
\text { tingencias y contingencias. }\end{array}$ & $\begin{array}{c}\text { Correspondencia ausente entre descripciones } \\
\text { de contingencias y contingencias. }\end{array}$ \\
2 (36 participantes). & $\begin{array}{c}\text { Correspondencia ausente entre descripciones } \\
\text { de contingencias y contingencias. }\end{array}$ & $\begin{array}{c}\text { Correspondencia entre descripciones de con- } \\
\text { tingencias y contingencias. }\end{array}$ \\
\hline
\end{tabular}


tarea. Las cuatro tareas siguientes se realizaban de manera similar dentro de los 45 minutos estipulados para la sesión.

Después de cada bloque se evaluó la conducta autocontrolada mediante una situación de elección. La situación fue la siguiente: si los participantes querían (de manera individual), podían realizar una actividad adicional que consistía en llenar una plana con letras por el lado anterior y posterior de una hoja, obteniendo así una consecuencia positiva. Para esto el experimentador proporcionó la siguiente descripción:

Hagamos una actividad más. Aquí tengo unas hojas que son como esta (indicando la plana), tienen una cuadrícula de lado y lado con letras al inicio. Lo que debe hacer cada uno de ustedes es llenar los dos lados completamente con las letras que aparecen al inicio. A la persona que lo haga, la dejo salir a jugar 15 minutos antes de entrar a la otra clase. Entonces quien quiera llenar la planilla, debe levantar la mano, venir a recoger la hoja y llenarla completamente.

\section{RESULTADOS}

Los resultados de este experimento indicaron que el comportamiento de elección en la evaluación de autocontrol de 23 de los 37 participantes que experimentaron los ensayos en el orden correspondencia-correspondencia ausente se modificó en función de cada bloque. Al presentarse correspondencia entre descripciones de contingencias y contingencias, los participantes eligieron realizar una tarea monótona con el fin de obtener un reforzador postergado de mayor magnitud. Por el contrario, cuando experimentaron ausencia de correspondencia en la situación experimental previa, se comportaron en función de consecuencias inmediatas.

En la misma secuencia de presentación de los bloques, 13 participantes mantuvieron su conducta en las dos mediciones de autocontrol, 12 se comportaron en función de consecuencias demoradas después de cada bloque de ensayos, y uno en función de consecuencias inmediatas en ambas mediciones.

En la misma situación, un participante mostró comportamiento bajo paradigma de autocontrol contrario a lo esperado, es decir, después de experimentar correspondencia entre descripciones y contingencias, en la tarea de autocontrol eligió la opción inmediata. Por el contrario, cuando experimentó ausencia de correspondencia, en la evaluación de autocontrol se comportó en función de consecuencias demoradas.

En la Figura 3 se ilustra la tendencia de elección durante la evaluación de autocontrol de los 37 participantes previamente descrita.

Por otra parte, los resultados indicaron que el comportamiento de elección durante la evaluación de autocontrol de 27 de los 36 participantes que experimentaron la secuencia de ensayos de correspondencia ausente-correspondencia se modificó en función de los mismos. Es decir, al experimentar ausencia de correspondencia entre descripción de contingencias y consecuencias, eligieron la opción inmediata; sin embargo, durante la sesión dos, cuando se presentó correspondencia entre la descripción dada por el experimentador y las contingencias, estos participantes eligieron la opción demorada durante la tarea configurada para evaluar autocontrol. De los participantes restantes,

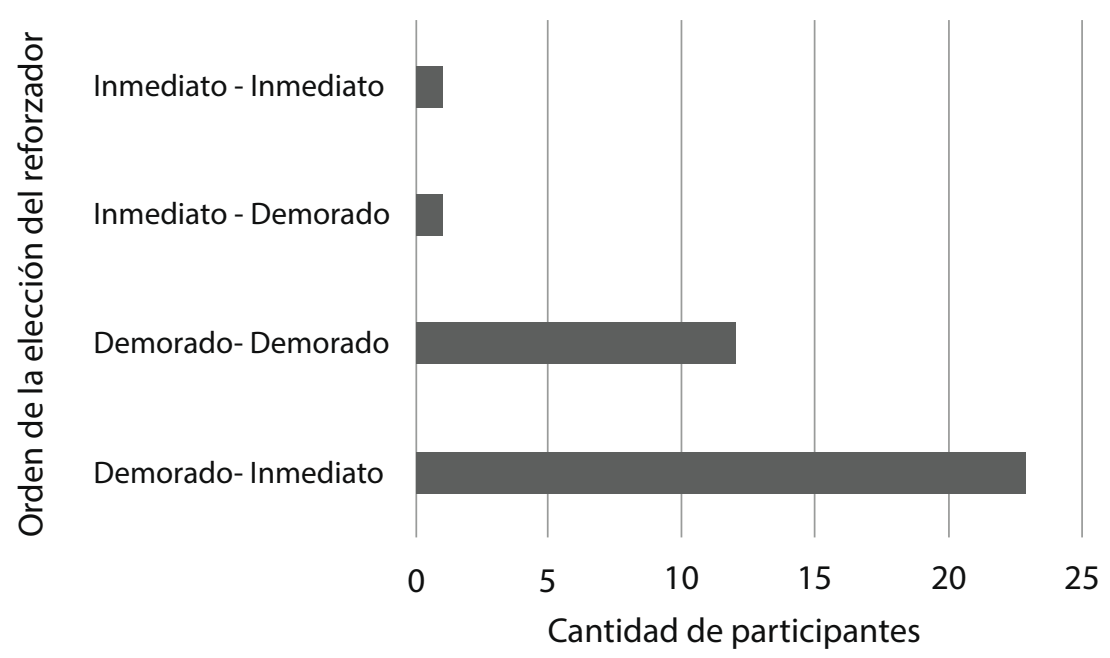

Figura 3. Conducta de elección en la condición experimental de correspondencia y correspondencia ausente. 
siete eligieron la opción demorada en las dos evaluaciones de autocontrol y uno mantuvo su conducta de elección en la opción inmediata.

Finalmente, un participante se comportó de manera contraria a lo esperado, es decir, al experimentar correspondencia ausente entre descripciones y contingencias, eligió la opción demorada; y cambió su elección a inmediata en la sesión dos, en la que experimentó correspondencia entre descripciones y contingencias. En la Figura 4 se muestra los resultados descritos anteriormente.

\section{DISCUSIÓN}

En los dos experimentos realizados, los participantes fueron expuestos a ensayos de correspondencia y ausencia de correspondencia entre descripciones de contingencias y contingencias, y se sometieron posteriormente a una tarea de elección bajo paradigma de autocontrol que no guardaba similitud formal o funcional con los ensayos antes mencionados — es decir, la elección del reforzador demorado o inmediato no fue entrenada de manera directa- .

Se observó que después de experimentar los ensayos de correspondencia entre descripciones de contingencias y contingencias, los participantes del estudio, en su mayoría, eligieron el reforzador demorado de mayor magnitud, lo que a la luz de la TMR podría implicar que respondieron ante un evento (situación de elección) en términos de otro (ensayos de correspondencia), mediante la abstracción de la función de una clave contextual de causalidad presente en las dos situaciones (Msetfi, Wade \& Murphy, 2013; Törneke et al., 2008; Valdivia \& Luciano, 2006). En otras palabras, podría asumirse que la descripción de la contingencia que configuraba la situación de elección para medir autocontrol incluía claves contextuales (si, entonces) que le permitieron al participante enmarcar su conducta y los eventos ambientales en relaciones de causalidad, lo que hizo posible que su comportamiento fuera controlado por consecuencias que probablemente solo serían experimentadas a largo plazo. De esta manera, la historia de aprendizaje establecida durante la exposición a los ensayos de correspondencia alteró las funciones de las descripciones de contingencias, incrementando su efectividad para vincular la consecuencia postergada con la tarea que permitiría contactarla (Gómez et al., 2007).

Este hallazgo es respaldado por Barnes-Holmes et al. (2005), quienes mencionan que el aprendizaje relacional es una respuesta operante flexible que no se desliga del control de las consecuencias. De hecho, antes de llegar a comportarse ante un evento en función de otro, es necesaria la repetición de múltiples ejemplares que permitan abstraer la función de la clave que los relaciona, la cual sigue siendo modificable por la experiencia.

En contraste, podría decirse que después de experimentar una historia de correspondencia ausente entre descripciones de contingencias y contingencias, el responder relacional se vio debilitado, impidiendo que la clave contextual contenida en la descripción controlara la conducta de elección de manera efectiva. Es decir, la historia de aprendizaje generada en esta situación afectó la efectividad de la descripción de contingencias para alterar los eventos referidos en ella, haciendo que, en su mayoría, los participantes evitaran realizar una tarea larga y monótona (transcripción de letras), aun cuando en la descripción que configuraba la situación de elección, esta era la condición para conseguir

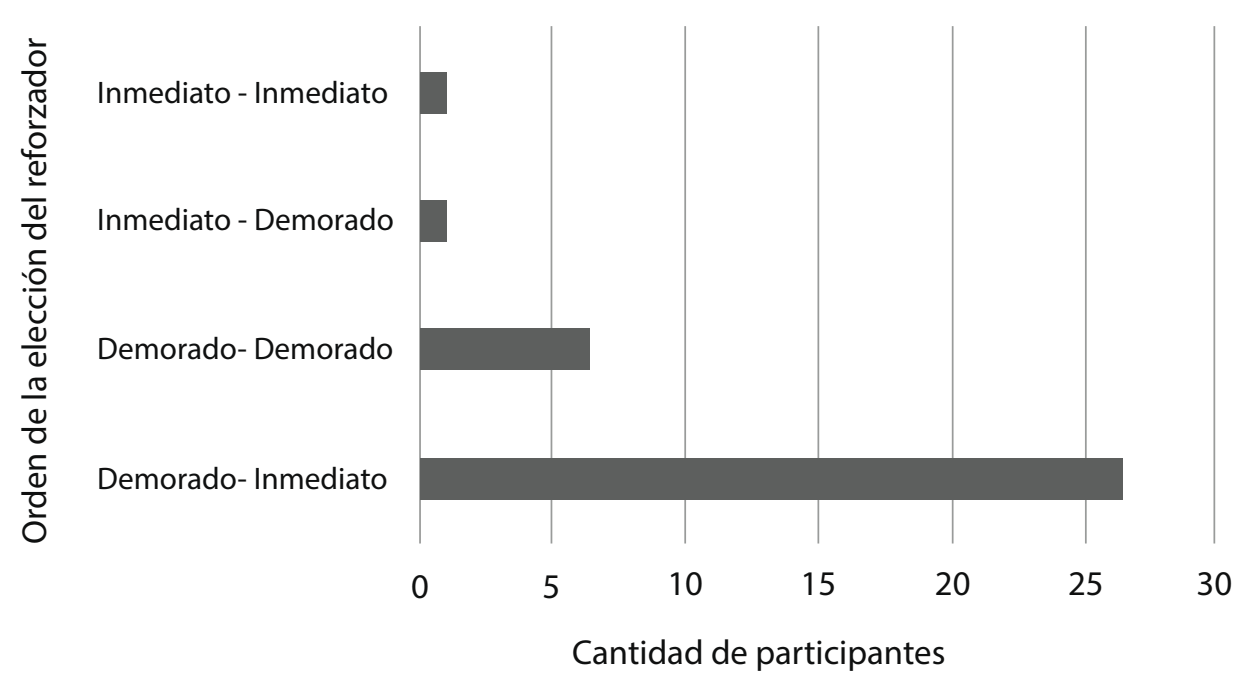

Figura 4. Conducta de elección en la condición experimental de correspondencia ausente y correspondencia. 
un reforzador de mayor magnitud. Como resultado, otras condiciones presentes - como el refuerzo inmediato- se hicieron más relevantes en la conducta de elección de los participantes (Gómez et al., 2007; Gómez \& Luciano, 2000).

Estos resultados se relacionan con hallazgos de estudios previos en los que se ha evidenciado que el proporcionar descripciones de contingencias verdaderas y reforzar de manera continua el ajuste conductual de los individuos ante las descripciones de contingencias favorece la efectividad del participante en tareas de discriminación condicional. Por el contrario, la falsedad de las descripciones, además de generar variabilidad en las ejecuciones, disminuye progresivamente el seguimiento de descripciones de contingencias e incrementa la probabilidad de comportarse bajo el control de las contingencias (Martínez, Ortíz \& González, 2007; Ortíz \& Cruz, 2011; Ortíz, González, Rosas \& Alcaraz, 2006).

Ahora bien, aunque la tendencia a elegir uno u otro reforzador según los ensayos experimentados se evidenció en la mayoría de participantes, se presentaron algunas excepciones. En el primer experimento, dos participantes eligieron el reforzador demorado en la evaluación de autocontrol independientemente de los ensayos de correspondencia experimentados - es decir, correspondencia o ausencia de correspondencia entre descripciones de contingencias y contingencias-, y otro mantuvo la elección del reforzador inmediato. $Y$ en el segundo experimento, 21 de los 73 participantes mantuvieron su conducta en las dos mediciones de autocontrol después de los dos tipos de ensayos, 19 en la opción demorada y dos en la opción inmediata. Este comportamiento - que no fue la tendencia general - sugiere que la historia pre-experimental de los participantes (variable que no estaba bajo el control de los investigadores), pudo haber interactuado con las condiciones experimentales de manera que no se alteraron las funciones ya adquiridas por la clave contextual "si, entonces".

Teniendo en cuenta que el aprendizaje relacional es una operante generalizada funcionalmente (Gómez et al., 2007), de estos resultados se puede inferir que al propiciar una historia de correspondencia a través de situaciones y fuentes variadas durante el desarrollo de un individuo, principalmente en la infancia - donde se aprende la capacidad de adaptarse a nuevas situaciones (Ruiz \& Gómez, 2010)—, es posible fortalecer la función de claves contextuales que faciliten el seguimiento de descripciones de contingencias y le permitan quedar bajo el control de su propio comportamiento verbal, favoreciendo así la elección de reforzadores demorados de mayor magnitud.

Esto es coincidente con los planteamientos de la Terapia de Aceptación y Compromiso, derivada de la TMR, a partir de la cual es posible postular que el comportamiento de los participantes se reguló a través de "pliance", un tipo de conducta gobernada por regla, en el que la función de una formula verbal se establece por una historia en la que las consecuencias están mediadas por otros (Hayes et al., 2001; Wilson \& Luciano, 2007). En este mismo sentido, el comportamiento de los participantes en quienes se evidenció variabilidad en la conducta de elección en la tarea de autocontrol fue facilitado por un "pliance flexible", en tanto les permitió ponerse en contacto con las contingencias que experimentaron durante las fases de correspondencia y ausencia de correspondencia entre descripciones de contingencias y contingencias, lo que generó que su elección durante la tarea de autocontrol se ajustara en función de la historia experimental en la opción demorada o inmediata.

En contraste, en los casos en que la conducta de elección no se presentó en la dirección esperada se plantea la posibilidad de que este comportamiento se haya presentado por los efectos de la regulación verbal que puede producir un "pliance rígido", lo que les dificultó a los participantes ponerse en contacto con las contingencias de las historias de correspondencia y ausencia de correspondencia. En estos casos, es probable que el comportamiento se regulara por las relaciones verbales que se establecieron previamente en la historia de aprendizaje del participante, relaciones que no se vieron alteradas por la historia experimental. Autores como Valdivia y Luciano (2006) explican este comportamiento afirmando que las palabras y los gestos pueden alterar las funciones de eventos en diferentes niveles y formas según la historia personal y los eventos que se sitúan en un momento determinado.

Por lo anterior, y asumiendo - desde el punto de vista de la TMR - que el individuo se comporta en el presente de acuerdo con las funciones aprendidas de manera directa y derivada a través de su historia (Luciano, Valdivia, Gutiérrez \& Páez, 2006), a partir de estos hallazgos se sugiere el estudio de otros factores individuales involucrados en el comportamiento de elección bajo paradigma de autocontrol, así como el desarrollo de investigaciones tendientes a explicar la variabilidad del comportamiento de los individuos en cuanto a las situaciones en las que se evidencia autocontrol; por ejemplo, cuando una persona presenta mayor tendencia a adoptar una dieta saludable y a realizar una rutina de ejercicio, pero pasa gran parte de su tiempo mirando televisión en lugar de realizar sus actividades escolares o laborales.

De igual forma, este estudio puede ser replicado con participantes de diferentes edades a fin de comprobar si el efecto de la variación experimental de la historia de correspondencia entre descripciones de contingencias y contingencias sobre la tendencia a elegir consecuencias inmediatas o demoradas varía en función de la edad. Esto teniendo en cuenta que las relaciones verbales que 
se establecen se vuelven más complejas a medida que se interactúa con situaciones novedosas o se modifican las funciones del lenguaje ya establecidas.

Para concluir, es preciso mencionar que estos resultados son preliminares, y antes de llevarlos al contexto aplicado, es necesario realizar otros experimentos que permitan confirmar la relación funcional entre el lenguaje y el autocontrol. Cabe resaltar que en esta publicación se muestran los resultados de un estudio en un contexto donde los mecanismos de control permiten aislar variables extrañas e incrementar la validez interna de los resultados - de laboratorio- (Kerlinger \& Lee, 2002) y otro, en un ambiente natural simulado, que si bien incrementa las probabilidades de afectar negativamente la validez interna, favorece la validez externa de las conclusiones extraídas. Todo lo anterior aporta de manera significativa a lo que para efectos de la ciencia y el contextualismo, en particular, es fundamental: la predicción y el control del comportamiento humano (Valdivia \& Luciano, 2006).

\section{REFERENCIAS}

Alós, F., Guerrero, M., Falla, D., \& Amo, A. (2013). Estímulos compuestos, discriminaciones simples y transferencia del aprendizaje en nuevas discriminaciones simples y condicionales. International Journal of Psychology and Psychological Therapy, 13(1), 97-112. Recuperado de http:/www. ijpsy.com/volumen13/num1/349/estmulos-compuestos-discriminaciones-simples-ES.pdf.

American Psychological Association. (2012). Lo que se necesita saber acerca de la fuerza de voluntad: la ciencia psicológica del autocontrol. Recuperado de http://www.apa. org/helpcenter/willpower-spanish.pdf.

Barnes-Holmes, D., Rodríguez, M., \& Whelan, R. (2005). La teoría de los marcos relacionales y el análisis experimental del lenguaje y la cognición. Revista Latinoamericana de Psicología, 37(2), 255-275. Recuperado de http://www. redalyc.org/articulo.oa?id=80537203.

Benabou, R., \& Tirole, J. (2004). Willpower and Personal Rules. Journal of Political Economy, 112(4), 848-887. Recuperado de http://www.princeton.edu/ rbenabou/papers/ JPE2004.pdf.

Binder, L., Dixon, M., \& Ghezzi, P. (2000). A procedure to teach self-control to children with attention deficit hyperactivity disorder. Journal of Applied Behavior Analysis, 33(2), 233-237. Recuperado de https:/www.ncbi.nlm.nih. gov/pmc/articles/PMC1284241/.

Cáceres, D., Salazar, I., Varela, M., \& Tovar, J. (2006). Consumo de drogas en jóvenes universitarios y su relación de riesgo y protección con los factores psicosociales. Universitas Psychologica, 5(3), 521-534. Recuperado de http:// www.scielo.org.co/pdf/rups/v5n3/v5n3a08.pdf.
Corral, V. (2006). Contribuciones del análisis de la conducta a la investigación del comportamiento pro-ecológico. Revista Mexicana de Análisis de la Conducta, 32(002), 111-127. Recuperado de http://redalyc.uaemex.mx/pdf/593/59332203. pdf.

Dixon, M., \& Cummings, A. (2001). Self-control in children with autism: response allocation during delays to reinforcement. Journal of Applied Behavior Analysis, 34 (4), 491495. Recuperado de http://www.ncbi.nlm.nih.gov/pmc/articles/PMC1284343/pdf/11800188.pdf.

Dixon, M., Rehfeldt, R., \& Randich, L. (2003). Enhancing tolerance to delayed reinforcers: The role of intervening activities. Journal of Applied Behavior Analysis, 36(2), 263266. Recuperado de http://www.ncbi.nlm.nih.gov/pmc/ articles/PMC1284440/pdf/12858992.pdf.

García, C. (2008). Riesgos del uso de internet por niños y adolescentes. Estrategias de seguridad. Acta Pediátrica México, 29(5), 273-279. Recuperado de http://nietoeditores. com.mx/download/actapediatrica/Sep-Oct2008/ActaPediat-273-9.pdf.

Gómez, I., \& Luciano, C. (1991). Autocontrol en niños: un estudio experimental sobre dos procedimientos en la adquisición de conductas de espera. Psicothema, 3(1), 25-44. Recuperado de http://www.psicothema.com/psicothema. asp?id=2002.

Gómez, I., \& Luciano, C. (2000). Autocontrol a través de reglas que alteran la función. Psicothema, 12(003), 418-425. Recuperado de http://redalyc.uaemex.mx/src/inicio/ArtPdfRed.jsp?iCve=72712314.

Gómez, M., López, F., \& Mesa, H. (2007). Teoría de los marcos relacionales: algunas implicaciones para la psicopatología y la psicoterapia. International Journal of Clinical and Health Psychology, 7(2), 491-507. Recuperado de http:// redalyc.uaemex.mx/redalyc/pdf/337/33717060015.pdf.

González, J., Ávila, R., Juárez, A., \& Miranda, P. (2011). ¿Es la "abstención" de comer comida disponible un ejemplo de conducta de autocontrol en palomas? Acta comportamentalia, 19(3), 255-267. Recuperado de http:// redalyc.uaemex.mx/src/inicio/VentanaResumenTabs. jsp?claveArt=27452012 9001 .

Grosch, J., \& Nuringer, A. (1981). Self-control in pigeons under the Mischel paradigm. Journal of applied behavior analysis, 35(1), 3-21. Recuperado dep:/www.ncbi.nlm.nih.gov/ pmc/articles/PMC1333016/.

Guevremont, D., Osnes, P., \& Stokes, T. (1986). Preparation for effective self-regulation: The development of generalized verbal control. Journal of Applied Behavior Analysis, 19(1), 99-104. Recuperado de http:/www.ncbi.nlm.nih.gov/pmc/ articles/PMC1308046/.

Hernández, A., \& Sandoval, M. (2003). La actividad simbólica humana: una revisión de las tendencias contemporáneas en el análisis del comportamiento verbal. Acta Colombiana de Psicología, 1(10), 73-87. Recuperado de http://www.redalyc.org/pdf/798/79801006.pdf. 
Kerlinger, F., \& Lee, H. (2002). Investigación del comportamiento ( $4^{\mathrm{a}} \mathrm{Ed}$.). México: McGraw-Hill.

Luciano, C., Gómez, S., Hernández, M., \& Cabello, F. (2001). Alcoholismo, evitación experiencial y Terapia de Aceptación y Compromiso (ACT). Análisis y Modificación de Conducta, 27(113), 333-371.

Luciano, C. (1992). La conducta verbal a la luz de recientes investigaciones. Su papel sobre otras conductas verbales. Psicothema, 4(2), 445-468. Recuperado de http://redalyc. uaemex.mx/src/inicio/ArtPdfRed.jsp?iCve=72704210.

Luciano, C., Valdivia, S., Gutiérrez, O., \& Páez, M. (2006). Avances desde la terapia de aceptación y compromiso (ACT). Edupsykhé, 5(2), 173-201. Recuperado de http:// www.ucjc.edu/pdf/publicaciones/edupsikhe/vol-5/cap3 vol5-2.pdf.

Martin, G., \& Pearl, J. (2006). Modificación de Conducta. Qué es y cómo aplicarla. Madrid: Prentice Hall.

Martínez, H., Ortíz, G., \& González, A. (2007). Efectos diferenciales de instrucciones y consecuencias en ejecuciones de discriminación condicional humana. Psicothema, 19(1), 14-22. Recuperado de http://dialnet.unirioja.es/servlet/ articulo? codigo $=2231448$.

Montgomery, W. (2008). Teoría, investigación y aplicaciones clínicas del autocontrol. Psicothema, 11(2), 215-225. Recuperado de http://www.scielo.org.pe/pdf/rip/v11n2/ a13v11n2.pdf.

Msetfi, R., Wade, C., \& Murphy, R. (2013). Context and Time in Causal Learning: Contingency and Mood Dependent Effects. PLoS ONE, 8(5), 1-15. Recuperado de http://www. readcube.com/articles/10.1371\%2Fjournal.pone.0064063.

Naciones Unidas (2012). Informe mundial sobre las Drogas, 2012. Nueva York: Oficina contra la Droga y el Delito. Recuperado de https://www.unodc.org/documents/data-andanalysis/WDR2012/WDR_2012_Spanish_web.pdf.

Organización Mundial de la Salud \& Ministerio de Sanidad y Consumo (2006). Tabaco. Mortifero en todas sus formas. Recuperado de http://www.who.int/tobacco/resources/publications/wntd/2006/translations/Brochure_Spanish.pdf.

Organización Mundial de la Salud (2010). 10 datos sobre medio ambiente y prevención de enfermedades. Recuperado de http://www.who.int/features/factfiles/environmental_ health/es/index.html.

Organización Mundial de la Salud (2012). Obesidad y sobrepeso. Recuperado de http://www.who.int/mediacentre/factsheets/fs311/es/index.html.

Ortiz, G., \& Cruz, Y. (2011). El papel de la precisión instruccional y la retroalimentación en la ejecución y descripciones poscontacto. Revista Mexicana de Análisis de la Conducta, 37(1), 69-87. Recuperado de http://www.redalyc.org/ pdf/593/59319102006.pdf.
Ortiz, G., González, A., Rosas, M., \& Alcaraz, F. (2006). Efectos de la precisión instruccional y la densidad de retroalimentación sobre el seguimiento, la elaboración y transmisión de descripciones en tareas de discriminación condicional. Acta Comportamentalia, 14(2), 103-130. Recuperado de http:// www.redalyc.org/articulo.oa?id=274520152001.

Ruiz, D., \& Gómez, I. (2010). Transformación de funciones: Marcos de coordinación y oposición de acuerdo con equivalencia. Revista Latinoamericana de Psicología, 42(2), 311-322. Recuperado de http://www.redalyc.org/articulo. oa? $\mathrm{id}=80515381011$.

Ruiz, D., \& Gómez, M. (2016). Papel del control instruccional en el estudio de las tendencias de regulación verbal. Universitas Psychologica, 15(2), 135-152. Recuperado de http://revistas.javeriana.edu.co/index.php/revPsycho/article/viewFile/11986/13902.

Sidman, M. (1971). Reading and auditory-visual equivalences. Journal of Speech and Hearing Research, 14, 5-13. Recuperado de http://jslhr.pubs.asha.org/article. aspx?articleid $=1749398$.

Skinner, B. (1981). Conducta verbal. México: Trillas.

Theler, R., \& Shefrin, H. (1981). An economic theory of selfcontrol. Journal of Political Economy, 89(2), 392-406. Recuperado de http://www.jstor.org/discover/10.2307/18333 17? uid $=3737808 \&$ uid $=2129 \&$ uid $=2 \& \quad$ uid=70\&uid=4\&s $\mathrm{id}=21101153672451$.

Törneke, N., Luciano, C., \& Valdivia, S. (2008). Comportamiento gobernado por reglas y problemas psicológicos. Journal of Psychology and Psychological Therapy, 8(2), 141-156. Recuperado de http://conductual- contextual.es/ files/7713/3881/5865/Torneke_Luciano_ValdiviaESPAOL. pdf.

Valdivia, S., \& Luciano M. (2006). Una revisión de la alteración de las propiedades reforzantes de los eventos en humanos. International Journal of Psychology and Psychological Therapy, 6(3), 425-444. Recuperado de http://www. redalyc.org/articulo.oa? $\mathrm{id}=56060310$.

Wechsler, D. (1981). WISC-R. Escala de inteligencia revisada para el nivel escolar. México: El Manual Moderno.

Wilson, K., \& Luciano, C. (2007). Terapia de aceptación y compromiso (ACT). Madrid, España: Pirámide.

Zentall, T., Galizio, M., \& Critchfied, T. (2002). Categoritazion, concept learning, and behavior analysis: an introduction. Journal of the Experimental Analysis Behavior, 78(3), 237248. Recuperado de https://www.ncbi.nlm.nih.gov/pmc/ articles/PMC1284898/. 